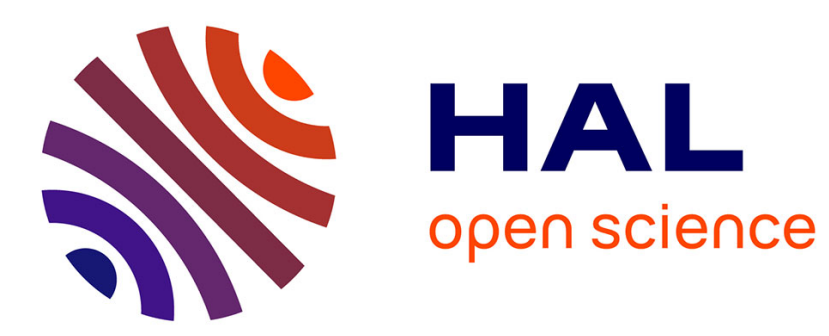

\title{
Emotional-related responses to critical events in problem solving
}

Evelyne Clément, Delphine Duvallet

\section{To cite this version:}

Evelyne Clément, Delphine Duvallet. Emotional-related responses to critical events in problem solving. Current Psychology Letters/Behaviour, Brain and Cognition, 2010, 26. hal-02004915

\section{HAL Id: hal-02004915 https://hal-cyu.archives-ouvertes.fr/hal-02004915}

Submitted on 2 Feb 2019

HAL is a multi-disciplinary open access archive for the deposit and dissemination of scientific research documents, whether they are published or not. The documents may come from teaching and research institutions in France or abroad, or from public or private research centers.
L'archive ouverte pluridisciplinaire HAL, est destinée au dépôt et à la diffusion de documents scientifiques de niveau recherche, publiés ou non, émanant des établissements d'enseignement et de recherche français ou étrangers, des laboratoires publics ou privés. 


\title{
Current psychology letters
}

Vol. 26, Issue 2, 2010 (2011)

Varia

Evelyne Clément and Delphine Duvallet

\section{Emotional-related responses to critical events in problem solving}

\begin{abstract}
Warning
The contents of this site is subject to the French law on intellectual property and is the exclusive property of the publisher.

The works on this site can be accessed and reproduced on paper or digital media, provided that they are strictly used for personal, scientific or educational purposes excluding any commercial exploitation. Reproduction must necessarily mention the editor, the journal name, the author and the document reference.

Any other reproduction is strictly forbidden without permission of the publisher, except in cases provided by legislation in force in France.
\end{abstract}

\section{revues.org}

Revues.org is a platform for journals in the humanites and social sciences run by the CLEO, Centre for open electronic publishing (CNRS, EHESS, UP, UAPV).

\author{
Electronic reference \\ cpl.revues.org/index5031.html \\ $\mathrm{DOI}$ : registration pending \\ Publisher: Centre PsyCLE \\ http://cpl.revues.org \\ http://www.revues.org \\ Document available online on: \\ http://cpl.revues.org/index5031.html \\ Document automatically generated on 10 novembre 2011. \\ (c) All rights reserved
}

Evelyne Clément and Delphine Duvallet, «Emotional-related responses to critical events in problem solving », Current psychology letters [Online], Vol. 26, Issue 2, 2010 | 2011, online since 10 novembre 2011. URL : http:// 


\section{Evelyne Clément and Delphine Duvallet}

\section{Emotional-related responses to critical events in problem solving}

We thank very much the two anonymous reviewers who accepted to expertise this study for their very relevant advices and their gratified comments on this paper.

\section{Introduction}

Although the greater part of the scientific community agrees with the idea of a strong interaction between emotion and cognition, surprisingly, in the framework of thinking and reasoning researches, emotions are still much neglected. The models and theories developed in a classical cognitive approach have allowed an increasingly fine understanding of human cognition like memory, perception, learning and reasoning. Yet, most of the cognitive models do not take into account the emotional component of a flexible cognition, which is a prerequisite condition for adaptation and evolution. However, it is well established that cognition and emotion are closely linked, and for instance, it was demonstrated that emotion modulates cognitive processes by driving selective attention (e.g. Öhman, Flykt, \& Esteves, 2001), guiding goals management (e.g. Oatley \& Johnson-Laird, 1987), allowing selection between alternatives (e.g. Bechara, Damasio, Damasio, \& Anderson, 1994; Damasio, 1994) and preparing the organism for action (Frijda, 1987). Conversely, it is postulated that the cognitive appraisal (interpretation) of the situation may trigger emotion (e.g. Scherer, Schorr, \& Johnstone, 2001).

\section{Cognition and emotion}

Nevertheless, this is not to say that emotion and cognition were not considered together at all, and that the study of their links is a new interest in the cognitive science framework. Actually, since four decades, some famous researchers in cognitive psychology have contributed to a highly important aspect of emotion theory by integrating it into a cognitive science framework. For instance, as far back as 1967 , Simon claimed the necessity to develop a general theory of thinking and problem-solving that incorporates motivation and emotion. Simon considered emotion and motivation as the two instances that control cognition by interrupting the system and allowing the processor to respond to urgent needs in real time. In the same line, Oatley and Johnson-Laird (1987) claimed that emotions have important cognitive functions and are central to the organization of cognitive processing. The authors proposed a theory in which "emotions have important cognitive functions, [...] they are part of management system to coordinate each individual's multiple plans and goals under constraints of time and other limited resources" (p.31).

Concurrently, following the pioneer works of Arnold (1960) and Lazarus (1966), cognitive theories of emotion came to light. The purpose of this theoretical field was to investigate the cognitive component of emotion. From this viewpoint, emotions are conceived as the result of a set of processes covering both the evaluation of the event according to its consequences for the well-being of the organism (appraisal) and the evaluation of the individual's adjustment potential (coping potential) according to the person's evaluation of his, her capacity to control the event and its consequences (e.g. Frijda, 1987; Lazarus, 1991; Scherer, 1984; Smith \& Lazarus, 1993). Despite some divergent approaches developed in the different cognitive theories of emotion, the protagonists came to an agreement about a set of appraisal criteria. These criteria include the perception of change in environment requiring deployment of attention (novelty, expectancy), the intrinsic pleasantness (valence, pleasantness), the relevance of the event to one's goals and needs (relevance, goal conduciveness, motive consistency), the attribution of agency and intention (responsibility, locus of causality), the evaluation of one's ability to cope with the event (controllability, power, potential coping) and the evaluation of the normative significance (internal and external standards). 


\section{Emotion and problem solving}

Surprisingly, in the scope of problem solving, there are few studies. On the one hand, relative to the study of the impact of emotion in problem solving, some studies, employing induction methods, focused on the influence of the affect on strategies. Results show that positive affect facilitates creative problem solving and leads to use top-down processing strategies and that negative affect leads to a more systematic processing of information and leads to use bottom-up processing strategies (Ashby, Isen, \& Turken, 1999; Fiedler, 2001). On the other hand, relative to the emotional-related responses observed during a high level activity, some researches are devoted to study of the physiological responses while participants are engaged in problem solving (for a review, see Pecchinenda, 2001). One attempt to test the physiological activity during problem solving is the research conducted by Pecchinenda and Smith (1996). In this study, the authors recorded the electrodermal activity while subjects were solving moderately and extremely difficult anagrams. Pecchinenda and Smith's data provide evidence for the positive correlation between the electrodermal activity and the coping-potential appraisal: the number of skin conductance responses and their amplitudes were greater when the participants appraised their coping potential as high (moderately difficult problems) than when they appraised it as low (extremely difficult problems). In a more recent research, van Reekum, Johnstone, Banse, Etter, Wehrle and Scherer (2004) aimed to study the physiological responses and the emotional self-report while participants were engaged in a video game. More precisely, in the framework of the Component Process Model of Scherer (see Scherer, 2001), the authors investigated two appraisal dimensions, intrinsic pleasantness and goal conduciveness of the game events. If one focuses on the physiological measures, results show that physiological responses were influenced by the goal conduciveness but not by the intrinsic pleasantness. Indeed, the skin conductance response magnitudes were higher for obstructive compared to conducive events, the acceleration of heart rate was observed in the obstructive events and the pulse transit time was shorter in these events than in the conducive events. Finally, muscle tension was higher for obstructive compared to conducive events.

On an aggregate, studies on the psychophysiological responses during problem solving show that both the difficulty of the task and the goal conduciveness influence the changes in physiological activity Appraisal theories postulate that these changes are determined by the personal interpretation of the situation through the appraisal process. In the line of this theoretical framework, changes in activity of the autonomic nervous system are interpreted as markers of the appraisal processes. Two kinds of appraisal processes are proposed to explain these changes. On the one hand, physiological changes are interpreted as concomitants of motivational and coping potential appraisals and may reveal task engagement/disengagement. Actually, in extremely difficult problem, participants appraised their coping potential as low and the situation as incongruent with their goal. On the other hand, changes in physiological activity are interpreted as concomitants of goal conduciveness appraisal: obstructive events involve the increase of the skin conductance responses' magnitude. In this case, electrodermal activity is interpreted as reflecting emotional arousal and an effortful allocation of attentional resources (Dawson, Schell, \& Filion, 2000).

\section{Purpose and hypotheses}

The purpose of the present study was to identify the patterns of emotional manifestations that are associated with critical events supposed to be emotionally relevant according to their conduciveness to performing well the problem. Because, as noted by Aue (2009), differences in the appraisals may exist between individuals, and consequently because it seems difficult to define a priori the conduciveness of an event, in the present research, and contrary to the method employed by van Reekum et al. (2004), the conduciveness of the events was not speculated a priori, but was identified on the basis of an individual protocol analysis. In order to reach this aim, the analysis consisted in conducting intra-subject comparisons between the different states of the problem which may be appraised as conducive or obstructive. Theses 
states were defined as the impasse situations and the subgoal-achievements as they are in the theoretical framework of Richard (Richard, Pointrenaud, \& Tijus, 1993).

In other respects, we were interested to investigate emotional-related responses associated to the two phases depicted by Kotovsky, Hayes and Simon (1985): the exploratory phase and the planning phase. In the exploratory phase, the participant learns how to make a move in order to reach the goal and is engaged in the construction of the relevant interpretation of the situation. In other words, this phase corresponds to the interpretation of the situation, and consists in exploring the problem-space in order to correctly apply move-operator observing the rules of the problem. In the planning phase, the adequate representation of the situation is constructed, the rules are integrated, and planning activities may occur. It is both the planning activity and the fast rhythm of the moves that permits to delimitate the two phases of problem solving (see for a detail description of the two phases, Clément \& Richard, 1997, Kotovsky, Hayes, \& Simon, 1985).

Further, although appraisal theories posit that the cognitive evaluations of the situation organize the emotional-related responses such as physiological and expressive behavior (see for a recent and synthetic presentation, Grandjean \& Scherer, 2009), little attention has been given to the concomitant physiological and expressive activity during problem solving. We postulate that facial expressions are well suited in the context of problem solving because facial expressiveness is generally considered as a component of emotion, a function of which is to communicate to someone his/her own internal states (Ekman, 1994; Izard, 1994; Kaiser $\&$ Wehrle, 2001). Moreover, it has been assumed that facial expressions reveal the person's appraisals and the action tendencies (Frijda, 1987) and, as Scherer and Ellgring (2007) wrote: "Facial expressions express components of emotion process, as patterned by the results of appraisal" (p.115). So that, the present study is a first attempt to examine both these two kind of emotional-related responses in problem solving.

Based upon the framework of the appraisal theories, some specific hypotheses may be formulated. On the one hand, in respect of the physiological component, if the skin conductance activity reflects the coping potential appraisals, it was predicted that the appraisal of an event as conducive (subgoal achievement) could increase the motivation, and the confidence would be indicated by the increase of the skin conductance responses. Conversely, if the skin conductance activity reflects emotional arousal and effortful resources allocation, the opposite pattern would be observed: the skin conductance responses would increase in the impasse situation. In respect of the comparison of the two phases of the problem solving, according to Pecchinenda and Smith, it was predicted an increase of the electrodermal activity in the planning phase and a decrease in the exploratory phase.

On the other hand, predictions for the expressive pattern were that the appraisal of event as conducive would be associated with positive facial expressions. Conversely, the appraisal of the event as obstructive (impasse situations) was predicted to be associated with negative facial expressions. In the same way, the comparison between the two phases of the problem solving would showed a greater number of positive facial expressions in the planning phase than in the exploratory phase and a greater number of negative facial expressions in the exploratory phase than in the planning phase.

These hypotheses were tested using a difficult problem-solving puzzle, the five disks version of the Tower of Hanoi problem. This relative difficult problem for adults was chosen because it was supposed to be difficult enough to motivate the participant to perform the task without exceeding his/her own objective ability to solve the problem.

\section{Method}

\section{Participants}

A total of 19 volunteer participants (mean age $=20.3$ years; $S D=5.26$ ) were recruited from the University of Rouen for their participation in the experiment. Because of the differences in physiological and expressive responses between females and males (Kring \& Gordon, 1998), only female participants were recruited. None of the participants knew the problem before the experiment. 


\section{The problem solving situation}

The five disks version of the Tower of Hanoi problem was presented under physical woody apparatus that was composed by a base containing three pegs and five disks of increasing size. The disks have holes in them so they can be stacked on the pegs. The disks all start out on the left peg, making a tower. The disks can be moved from any peg to any other peg. The goal was to move the tower from the left peg to the right one with respect to three rules: (1) only one disk may be moved at a time; (2) only the top disk on a peg can be moved; (3) a disk can never be placed on a smaller one. The optimal pathway to reach the goal is of 31 actions.

\section{Procedure}

After being informed of the aim of the study, all the participants of the present sample gave their informed consent. Participants were tested individually, in a quiet place of the laboratory, and were seated in a comfortable armchair. In a pre experimental session, sensors for the physiological measures were attached, and the skin conductance baseline was recorded at rest during one minute relaxation phase. Following the relaxation phase, the experimenter exposed instructions for performing the task and explicitly informed there was no limited time to perform it.In order to record the facial expressions and the actions they made to reach the goal, the participants were videotaped. A camera was placed at two meters of participants. At the end of the experimental session, a debriefing was given.

\section{Principles of the individual protocol analysis}

In order to identify the impasse situations and the subgoal achievements and to match the physiological responses and the facial expressions with the cognitive events, an individual protocol analysis was conducted in the framework of Richard's theory of problem solving. Based upon the recording of the behavioural (the sequences of the actions to reach the goal) and of the expressive and physiological data, a first analysis was conducted to identify the exploratory phase and the planning phase defined by Kotovsky et al. (ibid.). In the exploratory phase, the rhythm of actions is slow, many interruptions between actions and perseverative behaviours, lying in reiterating inefficient sequence of actions, are observed. In the planning phase, there is no interruption between actions, no perseveration, and the rhythm of actions is fast (see Figure 1 for an example of the end of the exploratory phase and the planning phase extracted from the protocol of a participant).

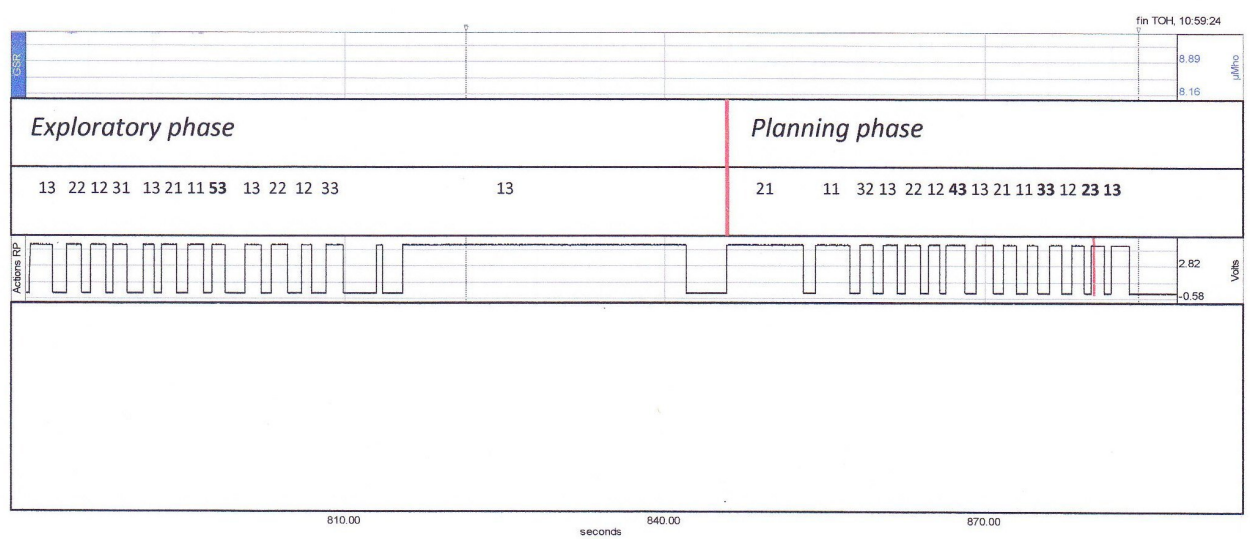

Figure 1. An example of the coding of the end of the exploratory phase and the planning phase (extracted from the protocol of a participant). All the actions were coded as following: the first digit represents the size of the disk (from 1 for the smallest to 5 for the largest disk), the second digit represents the current place ( 1 for the left, 2 for the middle, and 3 for the right). For example, 13 means that the smallest disk is placed on the right peg, 12 means that the smallest disk is placed on the middle peg, and 11 means that the smallest disk is placed on the left peg.

Within the exploratory phase, a second analysis was conducted between the impasse situations and the subgoal achievements. During this phase, the sequences of actions were recorded as 
an impasse or a subgoal achievement episode. Following Richard's definition, an interruption between two consecutive actions, a rule violation and a backward move (e.g. a move leading back to the previous state) were coded as an impasse episode. A subgoal achievement episode was defined by the fact that the participant reaches a state that achieves his/her current subgoal. For instance, the first subgoal which consists in placing the largest disk (5) on the right peg (3) by removing the smallest disks is rapidly achieved but apparently without planning. Actually, whereas the largest disk is on the right peg (3), the participant did not anticipate that the middle peg (2) has to be free in order to temporary place the disk (3) on it, and thus to put the disk (4) on the top of the largest disk (5). Instead of that, the participant put the disk (3) on the top of the disk (5). In this situation, the participant realized that it is impossible to place the disk (4) on the top of the disk (5), because of the disk (3), and that it is impossible to place the disk (3) elsewhere in the present configuration, This situation is what we defined as an impasse situation. To keep away from the impasse situation, it is necessary to construct a new subgoal: putting the disk (3) on the middle peg (2). Thus, participants takes a long time to make the first action in order to achieve this subgoal that is to say to put the smallest disk (1) on the right peg (3) in order to put the disk (2) on the left peg (1) (this example was extracted from the protocol of a participant).

When the adequate representation of the goal and of the means to reach the goal is constructed, then the sequence of the actions is optimal and rapidly executed. This breaking of execution rhythm and of the subgoal achievements determined the distinction between the exploratory and the planning phases.

\section{Collecting and coding data}

\section{Electrodermal activity}

The electrodermal activity was taken continuously throughout the problem solving. Skin conductance level (SCL) was measured using $\mathrm{Ag} / \mathrm{AgCl}$ electrodes (8mm diameter detection surface) filled with 0.05 molar $\mathrm{NaCl}$ electrode paste. The electrodes were placed on the palmer surfaces of the distal phalanges of second and third fingers of the non-dominant hand, and were secured by Velcro straps. A Biopac Advanced System was used to apply a constant 0.5 volt across the electrodes and to condition the skin conductance signal. This signal was sampled at $2 \mathrm{~Hz}$ through an analogue-to-digital interface and stored on the hard disk of a computer that faced the experimenter. One of the software tracks was used by the experimenter to index actions made by the participants, by pressing a press-button when the participant took a disk and when he put it on a peg (Figure 2 shows how the different data were recorded on line).

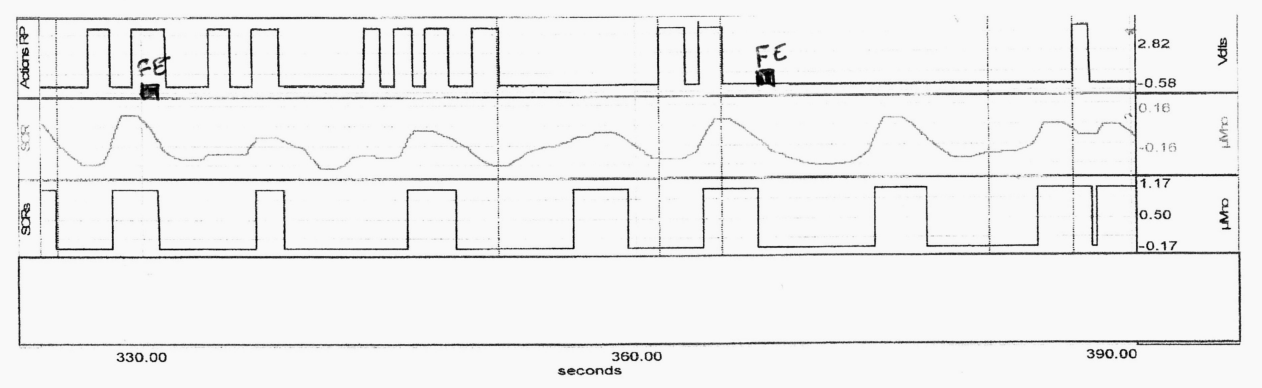

Figure 2. Physiological, expressive and behavioral data recorded on line. In the first track, both the actions made by the participant and the facial expressions were reported. In the second and third tracks, the skin conductance responses were recorded. The temporal dimension of the activity was reported on the abscissas axis.

Two measures of the skin conductance activity were computed for each period of interest. First, the number of spontaneous responses higher than $0.05 \mu$ Siemens initiated within the period of interest and expressed as a rate per minute (SCR-Rate). Then, the maximum amplitude (corresponding to pic-to-pic) of such responses was scored (SCR-MaxAm).

\section{Facial expressions}

Facial expressions of the participants were coded by an inter-judges method. The coding procedure was inspired by the inter-judges recognition of the FACES of Kring and Sloan 
(1991). Two judges, blind to the problem-solving activity, coded, in an independent way, the occurrence and the valence of the facial expressions. A facial expression was coded when the participant's face changes from a neutral state to a non-neutral state, and returns in a neutral state. The coders could not see the execution of the task (a mask was placed on the screen of the tube in such a way only the face of the participant was visible), and could not hear participant's talking (the coding was made without the sound). In order to be rigorous with only two coders, only the facial expressions which obtained a $100 \%$ inter-raters agreement were kept. The number of facial expressions per minute was computed both for the positive (PFE-rate) and the negative expressions (NFE-rate).

\section{Data analysis}

Two main analyses were conducted according to the periods of interest. The first analysis concerned the comparison between the exploratory and the planning phases; the second analysis was related to the comparison between the impasse situations and the subgoal achievements observed in the exploratory phase. Participants were specified as the subject variable, the two phases (exploratory phase/planning phase) and the events in the exploratory phase (impasse situations/subgoal achievement) were specified as the repeated variables. These periods were selected as factors and a fixed-effects model that included the main effects of these variables were specified. An alpha level of .05 was used for all statistical tests.

\section{Results}

In order to verify that the problem was sufficiently difficult for participants, we computed the global performance of the sample by using the following two classical indexes of performance in problem solving: the solution time and the number of actions to reach the goal. Both the mean solution time in minutes (mean $=9.61$; standard deviation $=5.91 ; \min =2.18 ; \max =25.73$ ) and the mean number of actions (mean $=81$; standard deviation= $31 ; \min =37 ; \max =135$ ) indicated that the problem was sufficiently difficult but without exceeding the objective ability of the participants to solve the problem (the optimal pathway to reach the goal is of 31 actions). Comparisons between the problem solving phases (exploratory/planning phase) and within the exploratory phase between the cognitive events (impasse situations/subgoal achievements) are presented in Table 1.

Table 1 Mean (and SDs) for each measure according to the periods of interest

\begin{tabular}{|l|l|l|l|l|l|l|l|l|}
\hline & \multicolumn{2}{l}{ Exploratory phase } & \multicolumn{2}{l|}{ Planning phase } & \multicolumn{2}{l|}{ Impasse situations } & \multicolumn{2}{l|}{ Subgoal achievements } \\
\hline & $\mathrm{M}$ & $(\mathrm{SD})$ & $\mathrm{M}$ & $(\mathrm{SD})$ & $\mathrm{M}$ & $(\mathrm{SD})$ & $\mathrm{M}$ & $(\mathrm{SD})$ \\
\hline SCR-rate & 5.55 & $(1.52)$ & 9.18 & $(2.45)$ & 5.33 & $(1.43)$ & 6.93 & $(1.53)$ \\
\hline $\begin{array}{l}\text { SCR- } \\
\text { MaxAm }\end{array}$ & 0.87 & $(0.30)$ & 0.87 & $(0.77)$ & 0.97 & $(0.55)$ & 0.73 & $(0.50)$ \\
\hline PFE-rate & 0.55 & $(0.49)$ & 3.42 & $(2.23)$ & 0.64 & $(0.69)$ & 0.38 & $(0.60)$ \\
\hline NFE-rate & 1.35 & $(0.81)$ & 0.56 & $(1.20)$ & 1.55 & $(1.31)$ & 0.73 & $(1.01)$ \\
\hline
\end{tabular}

Key: SCR-rate: skin conductance response ( $\mu$ Siemens/minute); SCR-MaxAm: maximum amplitude of the skin conductance responses; PFE-rate: positive facial expressions/minute, NFE-rate: negative facial expressions/minute.

\section{Emotional-related responses according to the problem solving phases}

Relative to the SCR-rate, the analysis showed significant main effect of problem solving phase, $t(18)=6.45 ; p=.00004$. As shown in Table 1, the SCR-rate was higher in the planning phase than in the exploratory phase. However, the differences of SCR-MaxAm of such responses were not significant, $t(18)<1$. Relative to the positive facial expressions, the analysis revealed significant effect of problem solving phase, the PFE-rate being higher in the planning phase than in the exploratory phase $(\mathrm{t}(18)=5.14 ; \mathrm{p}=.00006)$. In the same way, the analysis of the negative facial expressions showed main effect of the problem solving phase, the NFE-rate being higher in the exploratory phase than in the planning phase, $(\mathrm{t}(18)=2.14 ; \mathrm{p}=.04)$. 


\section{Emotional-related responses according to the impasse situations and subgoal achievements during the exploratory phase}

The comparison between the cognitive events during the exploratory phase showed main effect of Conduciveness of the events in the following way. Relative to the electrodermal activity, results showed that the SCR-rate was significantly higher in the subgoal achievements than in the impasse situations, $t(18)=3.99 ; p=.0008$, but the SCR-MaxAm of such responses was significantly higher in the impasse situations than in the subgoal achievements, $t(18)=$ 4.03; $p=.0007$. Relative to the positive facial expressions, whereas the PFE-rate was higher in the impasse situations than in the subgoal achievements, the analysis revealed no significant effect of the Conduciveness of the events $(t(18)=1.17 ; p=.25)$. Relative to the negative facial expressions, the NFE-rate was significantly higher in the impasse situations than in the subgoal achievements $(t(18)=2.19 ; p=.04)$.

In addition, complementary analyses, not directly concerned with the predictions, were conducted. Within-subject correlations were computed for each subject on the one hand across the performance and the physiological measures and on the other hand across the performance and the facial expressions.

Relative to the performance and the physiological measures, the analyses indicated that the solution time and the SCR-rate were significantly correlated ( $r=-0.63 ; \mathrm{p}=.003)$. In return, the solution time and the SCR-MaxAm were not significantly correlated ( $r=0.13 ; \mathrm{p}=.58)$. In the same way, the number of actions was correlated neither with the SCR-rate nor with the SCR$\operatorname{MaxAm}(r=0.36 ; \mathrm{p}=.13$ and $\mathrm{r}=0.38 ; \mathrm{p}=.10$, respectively).

Relative the performance and the facial expressions, none of the variables were significantly correlated. In order, correlations for solution time and PFE, solution time and NFE, number of actions and PFE, number of actions and NFE were $\mathrm{r}=-0.31 ; \mathrm{p}=.20 ; \mathrm{r}=-0.26, \mathrm{p}=.28 ; \mathrm{r}=0.15$, $\mathrm{p}=.52 ; \mathrm{r}=0.41, \mathrm{p}=.07$, respectively.

This set of results indicated that the faster solvers showed a greater SCR-rate than their slower counterparts. A more fine analysis showed that solution time and number of actions to reach the goal were not significantly correlated $(r=0.21 ; p=.37)$.

\section{Discussion}

The present paper aimed to investigate changes in the expressive behaviour and in the physiological activity during different appraisals associated with a problem solving situation. For this, while participants completed the five disks version of the Tower of Hanoi, electrodermal activity and facial expressions were recorded over the course of the activity. In order to match expressive, physiological, and behavioural data and, to test the changes in both physiological and expressive patterns, an individual protocol analysis was conducted.

First, if one focuses on the comparison between the cognitive events during the exploratory phase, our results partially support the hypothesis of Pecchinenda and Smith. Actually, our results showed that in the impasse situations the skin conductance rate was lower than in the subgoal achievements, and that the number of negative facial expressions was higher than in the subgoal achievement. Like this, these two emotional-related responses may be interpreted as reflecting the coping potential appraisals. In fact, it seems reasonable to think that achieving a subgoal increases the motivation to proceed with the problem and to appraise the ability to face the problem as high. In return, in the impasse situations, the estimated ability to face the problem might be low. The decrease of the skin conductance responses and the increase of the negative facial expressions observed in our research support this interpretation. However, with regard to the amplitude of the responses, the higher phasic skin conductance activity observed in response to the impasse situations is consistent with the interpretation that the amplitude of the responses reflects emotional arousal and an effortful allocation of attentional resources (Dawson, Schell, \& Filion, 2000).

This opposite and unpredicted pattern of the two electrophysiological measures, never reported in the literature, allows suggesting that the skin conductance response and the amplitude of this response do not underlie the same processes. In our research, we may suppose that the analysis of the dynamical dimension of the problem solving by collecting the physiological 
changes occurring during the successive events of the activity, makes possible to highlight this particular pattern of the electrodermal activity. Usually, in fact, the researches using the electrodermal activity do not conduct such an analysis. Namely, the changes of the electrophysiological activity are compared under different conditions (for example, under a difficult problem and an easy problem), or between the outcomes of the activity (the success or the failure).

Second, if one focuses on the comparison between the two phases of the problem solving socalled exploratory and planning phases, as predicted results showed that, on the one hand, the positive facial expressions rate and the skin conductance responses rate were significantly higher in the planning phase than in the exploratory phase, and on the other hand, the negative facial expressions rate was significantly higher in the exploratory phase than in the planning phase. This set of results is consistent with the hypothesis of Pecchinenda and Smith (1996) and the emotional-related responses chosen in our experiment may be interpreted as concomitants of motivation and coping potential appraisals. Actually, in the planning phase in which the adequate representation of the problem was built, and consequently in which the goal and the means to reach it have been understood, it seems reasonable to consider that participants appraised their coping potential as high even when, in the exploratory phase in which participants try to understand how to solve the problem and encounter difficulties, the coping potential would be evaluated as low.

In sum, the comparison between the two phases of the problem solving, showed a more positive facial expressions and electrodermal responses in the planning phase than in the exploratory phase, and on the other hand, more negative facial expressions in the exploratory phase. This first set of findings is consistent with the coping potential appraisals hypothesis. Positive facial expressions and the electrodermal responses may be interpreted as reflecting the motivation and the evaluation of a high capacity to face the problem in the planning phase. Relative to the comparison between the critical events in the exploratory phase, results showed more electrodermal responses in the subgoal achievements than in the impasse situations, whereas more negative facial expressions and higher amplitude of the electrodermal responses were observed in the impasse situations. This later pattern of results suggests that emotional arousal and effortful resources allocation are triggered in the impasse situations while participants appraise their capacity to face the problem as low.

Our findings showed the meaning of electrophysiological and expressive patterns of emotion in a new light. Furthermore, they urge to interpret those patterns with caution, especially in the studies that are aimed to assess the actual emotional state of a person.

Yet, some limitations and design of future studies have to be highlighted. Actually, in the present experiment, the coping potential appraisal was not directly assessed, but inferred on basis of the facial expressions and the skin conductance responses. Thus, in order to measure directly the individual differences in potential coping appraisal in impasse, some future experiments should be conducted. For instance, in an off-line processing, using verbal reports on the participants' subjective feeling while they had encountered impasse situations would convey direct information on the coping potential appraisal in these situations.

To conclude, this research was a first attempt to experimentally take into account the problem solving emotional component. Compared to more classical and passive induction methods (e.g. Ashby et al., 1999; Fiedler, 2001), we sought to identify emotional manifestations elicited while the participant is actively engaged in the task. Therefore, the events which are considering as relevant for emotion elicitation in the appraisal theories can be carefully manipulated and investigated in a relatively controlled setting.

\section{Bibliography}

Arnold, M. B. (1960). Emotion and personality: Psychological aspects (Vol. 1). New York: Columbia University Press.

Ashby, F. G., Isen, A. M., \& Turken, U. (1999). A neuropsychological theory of positive affect and its influence on cognition. Psychological Review, 106, 529-550. 
Aue, T. (2009). Psychophysiologie des émotions. In D. Sander \& K. R. Scherer (Eds.), Traité de psychologie des émotions (pp. 159-188). Paris: Dunod.

Bechara, A., Damasio A. R., Damasio, H., \& Anderson, S. W. (1994). Insensitivity to future consequences following damage to human prefrontal cortex. Cognition, 50, 7-15.

Clément, E., \& Richard, J-F. (1997). Knowledge of domain effects in problem representation: the case of Tower of Hanoi isomorphs. Thinking and Reasoning, 3(2), 133-157.

Damasio, A. R. (1994). Descartes' error: Emotion, reason, and the human brain. New York: Grosset Putnam.

Dawson, M. E., Schell, A. M., \& Filion, D. L. (2000). The electrodermal system. In J. T. Cacioppo, L. G. Tassinary, \& G. G. Bernstson (Eds.), Handbook of Psychophysiology,2nd ed. (pp. 200-223). New York: Cambridge University Press.

Ekman, P. (1994). Moods, emotions, and traits. In P. Ekman \& R. J. Davidson (Eds.), The nature of emotion: Fundamental questions (pp. 56-58). New York: Oxford UniversityPress.

Fiedler, K. (2001). Affective states trigger processes of assimilation and accommodation. In L. L. Martin $\&$ G. L. Clore (Eds.), Theories of mood and cognition: A user's guidebook. Mahwath, NJ: Erlbaum.

Frijda, N. (1987). Emotion, cognitive structure and action tendency. Cognition \& Emotion, 1, 115-143.

Grandjean, D., \& Scherer, K. R. (2009). Théorie de l'évaluation cognitive et dynamique des processus émotionnels. In D. Sander \& K. R. Scherer (Eds.), Traité de psychologie des émotions (pp. 43-76). Paris: Dunod.

Izard, C. E. (1994). Innate and universal facial expressions: Evidence from developmental and crosscultural research. Psychological Bulletin, 115, 288-299.

Kaiser, S., \& Wehrle, T. (2001). Facial expressions as indicators of appraisal processes. In K.R. Scherer, A. Schorr, \& T. Johnstone (Eds.), Appraisal processes in emotion:Theory, methods, research (pp. 285-300). New York: Oxford University Press.

Kotovsky, K., Hayes, J. R., \& Simon, H. A. (1985). Why are some problems hard? Evidence from Tower of Hanoi. Cognitive Psychology, 17, 248-294.

Kring, A.M., \& Gordon, A.H. (1998). Sex differences in emotion: expression, experience, and physiology. Journal of Personality and Social Psychology, 74(3), 686-703.

Kring, A.M., \& Sloan, D. (1991). The Facial Expression Coding System (FACES): A users guide. Unpublished manuscript.

Lazarus, R. S. (1966). Psychological stress and the coping process. New York: McGraw Hill.

Lazarus, R. S. (1991). Emotion and adaptation. New York: Oxford University Press.

Oatley, K., \& Johnson-Laird, P. N. (1987). Towards a cognitive theory of emotions. Cognition \& Emotion, 1(1), 29-50.

Öhman, A., Flykt, A., \& Esteves, F. (2001). Emotion drives attention: detecting a snake in the grass. Journal of Experimental psychology: General, 130(3), 466-478.

Pecchinenda, A. (2001). The psychophysiology of appraisals. In K. R. Scherer, A. Schorr, \& T. Johnstone (Eds.), Appraisal processes in emotion: Theory, methods, research (pp. 301-315). New York: Oxford University Press.

Pecchinenda, A., \& Smith, C. A. (1996). The affective significance of skin conductance activity during a difficult problem-solving task. Cognition \& Emotion, 10(5), 481-503.

Richard, J-F., Poitrenaud, S., \& Tijus, C.A. (1993). Problem- solving restructuration: Elimination of implicit constraints. Cognitive Science, 17, 497-529.

Scherer, K. R. (1984). On the nature and function of emotion: A component process approach. In K. R. Scherer \& P. Ekman (Eds.), Approaches to emotion (pp. 293-317). Hillsdale, NJ: Erlbaum.

Scherer, K. R. (2001). Appraisal considered as a process of multilevel sequential checking. In K. R. Scherer, A. Schorr, \& T. Johnstone (Eds.), Appraisal processes in emotion. Theory, methods, research (pp. 92-120). New York: Oxford University Press.

Scherer, K. R., \& Ellgring, H. (2007). Are facial expressions of emotion produced by categorical affect programs or dynamically driven by appraisal? Emotion, 7(1), 113-130.

Scherer, K. R., Schorr, A., \& Johnstone, T. (2001). Appraisal processes in emotion. Theory, Methods, Research. New York: Oxford University Press. 
Simon, H. A. (1967). Motivational and emotional controls of cognition. Psychological Review, 74(1), 29-39.

Smith, C. A., \& Lazarus, R. S. (1993). Appraisal components, core relational themes and the emotions. Cognition \& Emotion, 7, 233-269.

van Reekum, C. M., Johnstone, T, Banse, R., Etter, A., Wehrle, T., \& Schere K. R. (2004). Psychophysiological responses to appraisal dimensions in a computer game. Cognition \& Emotion, 18(5), 663-688.

\section{References}

Electronic reference

Evelyne Clément and Delphine Duvallet, «Emotional-related responses to critical events in problem solving », Current psychology letters [Online], Vol. 26, Issue 2, 2010 | 2011, online since 10 novembre 2011. URL : http://cpl.revues.org/index5031.html

\section{Evelyne Clément}

Université de Rouen Laboratoire de Psychologie et Neurosciences Cognitives (ICONES) 76821 Mont Saint Aignan Cedex France evelyne.clement@univ-rouen.fr Delphine Duvallet

Université de Rouen Laboratoire de Psychologie et Neurosciences Cognitives (ICONES) 76821 Mont Saint Aignan Cedex France

\section{Copyright}

(C) All rights reserved

\section{Abstract / Résumé}

The present paper aimed to investigate changes in the expressive behavior and in the physiological activity during different appraisals associated with a problem solving situation. To this purpose, while participants completed the five disks version of the Tower of Hanoi, electrodermal activity and facial expressions were recorded over the course of the activity. In order to match expressive, physiological, and behavioral data and, to test the changes in both physiological and expressive patterns, an individual protocol analysis was conducted. As predicted, results showed different patterns of expressive and physiological activity according to the events of the problem solving. Results are discussed in light of the goal conduciveness and the coping potential appraisals notions.

Keywords : problem solving, emotion, psychophysiology, facial expressions, individual protocol analysis

L'objectif de la présente étude est d'étudier les changements électrophysiologiques et les changements des expressions faciales associés aux évènements évalués comme émotionnellement pertinents pendant la résolution d'un problème. Pour atteindre cet objectif, l'activité électrodermale et les expressions faciales des participants ont été collectées tout au long de la résolution du problème de la Tour de Hanoi à 5 disques. Afin de synchroniser les actions des sujets et les changements physiologiques et expressifs, nous avons mené une analyse de protocoles individuels. Comme prédit, les résultats montrent différents patterns de l'activité électrophysiologique et expressive en fonction des évènements critiques de la résolution. Nous discutons les résultats à la lumière des notions d'obstruction de but et de potentiel d'ajustement issues des théories cognitives de l'émotion.

Mots clés : émotion, activité électrodermale, expressions facials, résolution de problèmes, protocoles individuels 